\title{
Mathematical Analysis of Effects of Surface Roughness on Steady Performance of Hydrostatic Thrust Bearings Lubricated with Rabinowitsch Type Fluids
}

\author{
U. P. Singh \\ Rajkiya Engineering College, Sonbhadra, Uttar Pradesh, India \\ Email: journals4phd@gmail.com
}

(Received June 27, 2019; accepted December 21, 2019)

\begin{abstract}
The objective of present theoretical analysis is to study the combined influence of surface roughness and lubricant inertia on the steady performance of stepped circular hydrostatic thrust bearings lubricated with nonNewtonian Rabinowitsch type fluids. To take the effects of surface roughness into account, Christensen theory of rough surface has been adopted. Solution for momentum equation has been derived by means of average inertia approach. Analytic expressions for film pressure have been established for radial and circumferential roughness patterns. Results for film pressure, load carrying capacity of bearing and lubricant flow rate has been plotted and analyzed on the basis of numerical results. Due to surface roughness, significant variations in these properties have been observed.
\end{abstract}

Keywords: Hydrostatic lubrication; Pressurized bearings; Rabinowitsch type fluids; Surface roughness; Thrust bearings.

\section{NOMENCLATURE}

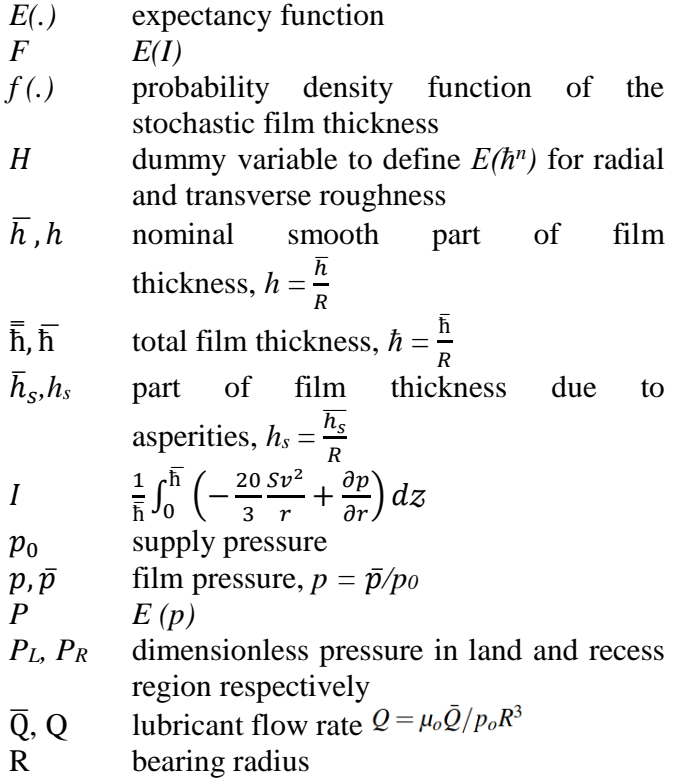

\section{INTRODUCTION}

Amongst the externally pressurized bearings, circular hydrostatic thrust bearings are of great importance for industrial and engineering

$$
\begin{array}{ll}
\overline{\mathrm{r}}, \mathrm{r} & \text { variable in radial direction, } r=\frac{\bar{r}}{R} \\
\bar{r}_{0}, \mathrm{r}_{\mathrm{o}} & \text { radius of supply hole, } r_{0}=\frac{\bar{r} \mathrm{0}}{R} \\
\bar{r}_{1}, \mathrm{r}_{1} & \text { step position, } r_{I}=\frac{\overline{r 1}}{R} \\
\mathrm{~S} & \frac{3}{20} \frac{\rho r^{2} \Omega^{2}}{P_{0}} \text { (inertia parameter) } \\
\bar{u}, u & \text { radial velocity, } u=\frac{\bar{u}}{R \Omega} \\
\bar{v}, v & \text { circumferential velocity, } v=\frac{\bar{v}}{R \Omega_{\bar{W}}} \\
\bar{W}, W & \text { load carrying capacity, } W=\frac{2 \pi R^{3} p_{o}}{R} \\
\bar{z}, z & \text { variable in } \mathrm{Z}-\text { direction, } z=\frac{\bar{z}}{R} \\
\alpha & \kappa p_{0}^{2} \\
\beta & \text { film thickness ratio } \\
\delta & \frac{p_{0} \mu_{0}}{\Omega} \\
\kappa & \text { coefficient of pseudoplasticity } \\
\mu & \text { viscosity } \\
\bar{\mu}, \mu & \text { viscosity of fluid, } \mu=\frac{\mu}{\mu_{0}} \\
\bar{\tau}_{r z}, \tau_{r z} & \text { shear stress, } \tau_{r z}=\frac{\tau_{r z}^{-}}{p_{o}}
\end{array}
$$

applications due to its wide uses in high speed rotating machines. Researchers have also paid significant attention towards improvement of performance of these bearings under various operating and lubricating conditions (Singh et al. 
2011b; Singh et al. 2013a; Singh et al. 2012c; Hamrock et al. 2004; Bassani and Piccigallo 1992). Several investigations have been carried out to analyse the dependence of different performance properties of these bearings on rotational inertia, fluid compressibility and temperature variation of the fluid (Coombs and Dowso 1964; Peterson et al. 1994; Kapur and Verma 1979). Optimization of design of hydrostatic thrust bearings with special consideration on shape and radii of recess and supply hole, and different operating conditions have been done time-to-time (Singh et al. 1983; Sharma et al. 2002; Tian et al. 2018; Younes 1993; Bakker and van Ostayen 2010; Sawano et al. 2015). Yadav and Kapur (1981) considered the simultaneous effects of temperature and rotational inertia of fluid on the overall performance of hydrostatic step thrust bearings. Some investigators also focused their attention to analyse the dynamic performance of hydrostatic thrust bearings lubricated with couple stress fluids (Zhicheng et al. 1993; Lin 1999).

On the same time, many researchers also emphasized that roughness of lubricated surfaces plays very important role on the performance of bearings (Christensen 1969). Prakash and Tiwari (1985) presented the analysis of porous bearing with surface corrugations. Singh et al. (1993) studied the characteristics of corrugated thrust bearings. Lin (2000) analysed the dynamic stiffness and damping of hydrostatic thrust bearings with corrugated surfaces. Yacout (2008) studied the effects of centripetal inertia on spherical shaped hydrostatic thrust bearings with rough walls. Xuebing et al. (2009) investigated the effects of surface toughness and rotational inertia on performance characteristics of high speed hydrostatic thrust bearings. Walicka et al. (2014) considered Ellis fluids model to analyse various performance characteristics of thrust bearing with rough walls.

Researchers also paid attention towards stabilizing the performance of industrial and commercial lubricants. It was observed that viscosity index of lubricants can be improved by blending high molecular weight polymer solutions to them (Stokes 1966; Wada and Hayashi 1971; Spikes 1994). Theoretical studies of different lubrication regimes with such additive based lubricants have been done with different non-Newtonian fluid models. Some of such models are Casson, Ellis, micropolar, powerlaw and couple stress models. Rabinowitsch or cubic stress model (Singh et al. 2011b; Singh et al. 2013a; Singh et al. 2012c) is one of the best fluid models to describe viscosity dependent characteristics of fluids. Wada and Hayashi (1971) showed with an experimental verification that this model accurately fits viscosity data of lubricants blended with additives. For one-dimensional fluid flow, Rabinowitsch fluid model is defined as following empirical relation:

$\bar{\tau}_{r z}+\kappa \bar{\tau}_{r z}^{3}=\bar{\mu} \frac{\partial \bar{u}}{\partial \bar{z}}$

where $\bar{\mu}$ is viscosity of fluid at zero shera rate, $\kappa^{-}$ coefficient of pseudoplasticity is responsible for nonNewtonian behaviour of fluids. This model works for Newtonian fluids $(\kappa=0)$, dilatant fluids $(\kappa<0)$ and pseudoplastic fluids $(\kappa>0)$. In the present decade, this model has been one of the frequently used fluid models for theoretical study of bearings (Bourging and Gay 1984; Hayashi and Wada 1974; Hashimoto and Wada 1986; Lin 2001). Singh et al. (2011a) presented theoretical analysis of pressurized flow between two curvilinear surfaces of revolution. Lin (2012) studied the characteristics of In the present investigation, it is proposed to uncover the combined influences of surface roughness, fluid inertia and non-Newtonian fluids on the performance properties of a hydrostatic thrust bearing using Rabinowitsch fluid model squeeze films between parallel annular plates. Singh et al. (2012b), Singh et al. (2012a), Singh et al. (2013b) investigated influences of nonNewtonian pseudoplastic lubricants on the various performances of squeeze films and hydrodynamic sliders. Singh et al. (2017), Singh et al. (2018) and Bhatt et al. (2017) also extended the applications of this fluid model to study peristaltic flow regimes of physiological fluids. Recently, Walicka et al. (2017b), Walicka et al. (2017a) used this model for theoretical investigation of film characteristics between two curvilinear rough surfaces. In light of this discussion, it is observed that type of lubricants and surface roughness are important considerations for theoretical prediction of bearings properties, life and stability. But none of the researchers has involved Rabinowitsch fluid model in theoretical analysis of hydrostatic thrust bearings, considering these aspects (surface roughness, non-Newtonian lubricant and fluid inertia) altogether.

In the present investigation, it is proposed to uncover the combined influences of surface roughness, fluid inertia and non-Newtonian fluids on the performance properties of a hydrostatic thrust bearing using Rabinowitsch fluid model.

\section{Constitutive Equations}

A schematic configuration of circular plates hydrostatic step thrust bearing with rough surfaces is shown in Fig.1. It is assumed that the lubricant is incompressible and non-Newtonian, the body couples and forces are not present, and usual assumptions of hydrodynamic lubrication are applicable to lubricant film. Under these assumptions, the governing equations for one dimensional, axially symmetric fluid flow in hydrostatic circular thrust bearings in dimensionless form ( $c f$. Appendix $-A$ ), together with constitutive Eq. (1) can be written as:

$\tau_{r z}+\alpha \tau_{r z}^{3}=\mu \frac{\partial u}{\partial z}$

$-\frac{20}{3} S \frac{v^{2}}{r}+\frac{\partial p}{\partial r}=\frac{\partial \tau_{r z}}{\partial z}$

$0=\mu \frac{\partial^{2} v}{\partial z^{2}}$

$\frac{1}{r} \frac{\partial}{\partial r}(r u)=0$

These equations satisfy the boundary conditions $u=$ 0 at $z=0, \beta \bar{\hbar} ; v=0$ at $z=0$ and $v=r \Omega$ at $z=\beta \bar{\hbar}$; $p\left(r_{o}\right)=1, p(1)=0$. The lubricant film thickness is 
taken as $\bar{\hbar}=\beta h+h_{s}$, where $h$ is the nominal smooth part of the film thickness, $\beta$ is step ratio, $h_{s}$ is the part of film thickness due to asperities on the surface measured from smooth level. In case of radial roughness, $h_{s}=h_{s}(\theta, \xi)$, and in case of transverse or circumferential roughness, $h_{s}=h_{s}(r, \xi)$; where, $\xi$ is a random variable to characterize some definite arrangement of the surface asperities.

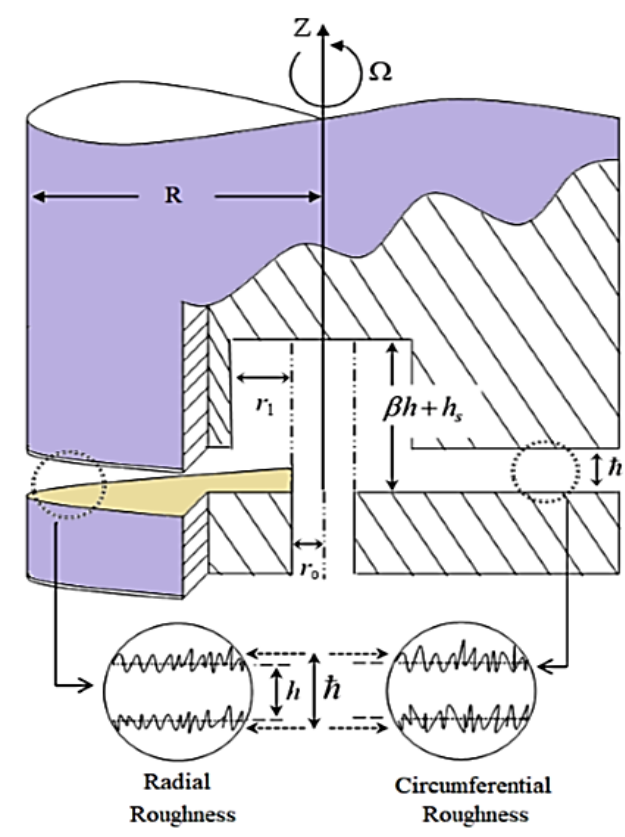

Fig. 1. Schematic diagram of step thrust bearing with rough surfaces.

\section{SOLUTION BY AVERAGE INERTIA THEORY}

From Eq.(4) and relevant boundary conditions, the circumferential velocity can be obtained as $v=$ $r z / \bar{\hbar}$ Now, taking the average of inertia term on the left hand side of radial momentum Eq. (3) across the film thickness and defining $I=\frac{1}{\bar{h}} \int_{0}^{\bar{h}}\left(-\frac{20}{3} \frac{s v^{2}}{r}+\right.$ $\left.\frac{\partial p}{\partial r}\right) d z=-\frac{20}{3} S r+\frac{\partial p}{\partial r}$, solution to Eq. (3) is obtained as $\tau_{r z}=I z+c_{l}$. Substituting this expression for $\tau_{r z}$ in Eq. (2) and integrating with the boundary conditions, a close form solution to the radial velocity $u$ can be obtained as:

$u=\frac{\delta}{2 \mu}\left[\begin{array}{c}I\left(z^{2}-z \beta \bar{\hbar}\right)+\alpha I^{3} \\ \times\left(\frac{z^{4}}{2}-z^{3} \beta \bar{\hbar}+\frac{3}{4} z^{2} \beta^{2} \bar{\hbar}^{2}-\frac{1}{4} z \beta^{3} \bar{\hbar}^{3}\right.\end{array}\right]$

Volumetric-flow rate of lubricant is defined as $\bar{Q}=$ $2 \pi \int_{0}^{\bar{h}} \bar{r} \bar{u} d \bar{z}$ to satisfy the equation of continuity, Eq. (5). Taking dimensionless form for flow rate $Q=$ $\mu_{0} \bar{Q} / p_{0} R^{3}$, a cubic relation between $Q$ and $I$ is obtained as follows:

$\bar{\hbar}^{3} I+\frac{3}{20} \alpha \bar{\hbar}^{5} I^{3}=-\frac{6 \mu Q}{\pi r}$

Taking the probability density function of the stochastic film thickness $f\left(h_{s}\right)$, (Christensen 1969): $f\left(h_{s}\right)=\left\{\begin{array}{l}\frac{35}{32 c^{7}}\left(c^{2}-h_{s}^{2}\right)^{3} ;-c<h_{s}<c \\ 0 ; \text { elsewhere }\end{array}\right.$

and defining the expectancy function $E(\boldsymbol{\Theta})$ as :

$\mathrm{E}(\Theta)=\int_{-\infty}^{\infty} \Theta f\left(h_{s}\right) d h_{s}$

stochastic form of Eq. (7) can be written as ( $c f$. Appendix -B):

$\mathrm{E}\left(\mathrm{H}^{3}\right) F=-\frac{6 \mu Q}{\pi r}+\frac{3}{20} \alpha \mathrm{E}\left(\mathrm{H}^{5}\right) F^{3}$

where, $E(I)=F ; \mathrm{E}\left(\mathrm{H}^{\mathrm{n}}\right)=\mathrm{E}\left(\bar{h}^{n}\right)$ for radial roughness and $\mathrm{E}\left(\mathrm{H}^{\mathrm{n}}\right)=\frac{1}{\bar{h}^{-n}}$ for circumferential roughness; $c$ is the half range assumed by the random film thickness variable $\xi$ with the standard deviation $\sigma$. The function $f\left(h_{s}\right)$ - defined in Eq. (8) terminates at $c=3 \sigma$. In the present analysis, $\mathrm{c}$ will be referred to as roughness parameter.

As Eq. (10) is nonlinear in $F$, it is not easy to solve it for analytical solution of pressure. Therefore, perturbation method is adopted to simplify it. Observing that the effective coefficient of $F^{3}$ is sufficiently smaller than 1 , considering $F=F_{o}+\alpha F_{l}$ will suffice for further analysis and, therefore, Eq. (10) can be simplified to

$F=-\frac{6 \mu Q}{\pi r E\left(H^{3}\right)}+\frac{3}{20} \alpha \frac{E\left(\mathrm{H}^{5}\right)}{E\left(H^{3}\right)}\left(\frac{6 \mu Q}{\pi r E\left(H^{3}\right)}\right)^{3}$

Noting that $F=E(I)=-\frac{20}{9} S r+\frac{\partial E(p)}{\partial r}$ from Eq.(34) and representing $E(p)=P$, expression for pressure is obtained from Eq.(11) as:

$P(r)=\frac{10}{9} S r^{2}-\frac{6 \mu Q}{\pi E\left(H^{3}\right)} \log r$

$-\frac{54}{4} \frac{\alpha \mu^{3} Q^{3}}{\pi^{3}} \frac{E\left(H^{5}\right)}{\left[E\left(H^{3}\right)\right]^{4}} \frac{1}{r^{2}}+c_{1}$

where, $c_{l}$ is the constant of integration.

Using the boundary conditions for pressure, $P\left(r_{o}\right)=$ $E\left(p\left(r_{o}\right)\right)=E(1)=1$ and $P(1)=E(p(1))=E(0)=0$ with the additional condition of continuity of pressure at the step, expression for pressure in recess region $\left(P_{R}\right)$ and land region $\left(P_{L}\right)$ are obtained as:

$$
\begin{aligned}
& P_{R}(r)=1+\frac{10}{9} S\left(r^{2}-r_{0}^{2}\right)-\frac{6 \mu Q}{\pi \mathrm{E}\left(H^{3}\right)} \log \left(\frac{r}{r_{0}}\right) \\
& -\frac{54}{4} \frac{\alpha \mu^{3} Q^{3}}{\pi^{3}} \frac{E\left(H^{5}\right)}{\left[E\left(H^{3}\right)\right]^{4}}\left(\frac{1}{r^{2}}-\frac{1}{r_{0}^{2}}\right) \\
& P_{L}(r)=\frac{10}{9} S\left(r^{2}-1\right)-\frac{6 \mu Q}{\pi \mathrm{E}\left(H^{3}\right)} \log r \\
& -\frac{54}{4} \frac{\alpha \mu^{3} Q^{3}}{\pi^{3}} \frac{\mathrm{E}\left(\mathrm{H}^{5}\right)}{\left[\mathrm{E}\left(H^{3}\right)\right]^{4}}\left(\frac{1}{r^{2}}-1\right)
\end{aligned}
$$

\subsection{Load Capacity and flow Rate}

Expression for lubricant flow rate $(Q)$ is derived in Appendix $-C$. The load capacity of bearing is defined as $W_{0}=\int_{0}^{R} \bar{r} \bar{P}(\bar{r}) d \bar{r} \quad$. Taking $W=\frac{\bar{W}}{2 \pi R^{3} p_{0}}$, dimensionless load capacity can be calculated as:

$W=\int_{0}^{1} r P(r) d r$

$=r_{0}^{2}+2 \int_{0}^{r_{1}} r P(r) d r+2 \int_{r_{1}}^{1} r P(r) d r$ 


\section{PRESSURE DISTRIBUTION}

\subsection{Case I: Radial Roughness}

For the radial roughness, the stochastic film thickness is $\bar{\hbar}=\beta h+h_{s}(\theta, \xi)$ and expressions for pressure in recess and land regions are:

$$
\begin{aligned}
& P_{R}(r)=1+\frac{10}{9} S\left(r^{2}-r_{0}^{2}\right)-\frac{6 \mu Q}{\pi \mathrm{E}\left(\bar{\hbar}^{3}\right)} \log \left(\frac{r}{r_{0}}\right) \\
& -\frac{54}{4} \frac{\alpha \mu^{3} Q^{3}}{\pi^{3}} \frac{\mathrm{E}\left(\bar{\hbar}^{5}\right)}{\left[\mathrm{E}\left(\bar{\hbar}^{3}\right)\right]^{4}}\left(\frac{1}{r^{2}}-\frac{1}{r_{0}^{2}}\right)
\end{aligned}
$$

where, $\mathrm{E}\left(\overline{\mathrm{h}}^{3}\right)=h^{3} \beta^{3}+\frac{c^{2} h \beta}{3}$ And $\mathrm{E}\left(\bar{\hbar}^{5}\right)=h^{5} \beta^{2}+$ $\frac{5 c^{4} h \beta}{33}+\frac{10 c^{2} h^{3} \beta^{3}}{9}$.

$P_{L}(r)=\frac{10}{9} S\left(r^{2}-1\right)-\frac{6 \mu Q}{\pi \mathrm{E}\left(\bar{\hbar}^{3}\right)} \log r$

$-\frac{54}{4} \frac{\alpha \mu^{3} Q^{3}}{\pi^{3}} \frac{\mathrm{E}\left(\bar{\hbar}^{5}\right)}{\left[\mathrm{E}\left(\bar{\hbar}^{3}\right)\right]^{4}}\left(\frac{1}{r^{2}}-1\right)$

where, $\mathrm{E}\left(\overline{\mathrm{h}}^{3}\right)=h^{3}+\frac{c^{2} h}{3} \quad$ And $\quad \mathrm{E}\left(\bar{\hbar}^{5}\right)=h^{5}+$ $\frac{5 c^{4} h}{33}+\frac{10 c^{2} h^{3}}{9}$.

\subsection{Case II: Circumferential or Circumferential Roughness}

For the radial roughness, the stochastic film thickness is $\bar{h}=\beta h+h_{s}(\theta, \xi)$ and expressions for pressure in recess and land regions are:

$$
\begin{aligned}
& P_{R}(r)=1+\frac{10}{9} S\left(r^{2}-r_{0}^{2}\right)-\frac{6 \mu Q A_{1}}{\pi} \log \left(\frac{r}{r_{0}}\right) \\
& -\frac{54}{4} \frac{\alpha \mu^{3} Q^{3}}{\pi^{3}} \frac{A_{1}^{4}}{A_{1}}\left(\frac{1}{r^{2}}-\frac{1}{r_{0}^{2}}\right)
\end{aligned}
$$

where,

$A_{1}=\mathrm{E}\left(\bar{\hbar}^{-3}\right)$

$=\frac{35}{32 c^{7}}\left[3\left(6 c^{2} h^{2} \beta^{2}-c^{4}-5 h^{4} \beta^{4}\right)\right.$

$\times \log \frac{(h \beta+c)}{(h \beta-c)}+30 c h^{3} \beta^{3}-26 c^{3} h \beta$

$A_{2}=\mathrm{E}\left(\bar{\hbar}^{-5}\right) \quad=\frac{35 / 32}{c^{7}\left(c^{2}-h^{2} \beta^{2}\right)}\left[3\left(c^{4}-6 c^{2} h^{2} \beta^{2}+\right.\right.$

$\left.\left.5 h^{4} \beta^{4}\right) \times \log \frac{(h \beta+c)}{(h \beta-c)}-30 c h^{3} \beta^{3}+26 c^{3} h \beta\right]$

and,

$$
\begin{aligned}
& P_{L}(r)=\frac{10}{9} S\left(r^{2}-1\right)-\frac{6 \mu Q B_{1}}{\pi} \log r \\
& -\frac{54}{4} \frac{\alpha \mu^{3} Q^{3}}{\pi^{3}} \frac{B_{1}^{4}}{B_{2}}\left(\frac{1}{r^{2}}-1\right)
\end{aligned}
$$

where,

$$
\begin{aligned}
& B_{1}=\mathrm{E}\left(\bar{\hbar}^{-3}\right) \\
& =\frac{35}{32 c^{7}}\left[3\left(6 c^{2} h^{2}-c^{4}-5 h^{4}\right) \log \frac{(h+c)}{(h-c)}\right. \\
& \left.+30 c h^{3}-26 c^{3} h\right] \\
& B_{2}=\mathrm{E}\left(\bar{\hbar}^{-5}\right) \\
& =\frac{\frac{35}{32}}{c^{7}\left(c^{2}-h^{2}\right)}\left[3\left(c^{4}-6 c^{2} h^{2}+5 h^{4}\right)\right. \\
& \times \log \frac{(h+c)}{(h-c)}+26 c^{3} h-30 c h^{3}
\end{aligned}
$$

such that $A_{1}=\frac{1}{h^{3} \beta^{3}}, A_{2}=\frac{1}{h^{5} \beta^{5}}, B_{1}=\frac{1}{h^{3}}$ and $B_{2}=$ $\frac{1}{h^{5}}$ when $\mathrm{c}=0$.

\section{RESULTS AND DISCUSSION}

In order to analyze the effect of roughness on the performance properties of externally pressurized thrust bearings lubricated with non-Newtonian (pseudoplastic) lubricants, pressure and load capacity have been plotted and compared for roughness parameter $c(0 \leq c \leq 0.005)$ and pseudoplasticity parameter $\alpha\left(\alpha=\kappa P_{0}^{2}\right)$. In order to provide a practical justification to this analysis, values of $\kappa$ have been taken from experimental work. $\kappa=5.65 \times 10^{-6} \mathrm{~m}^{4} / \mathrm{N}^{2}$ and $3.5 \times 10^{-6} \mathrm{~m}^{4} / \mathrm{N}^{2}($ Wada and Hayashi 1971). The values of operating parameters, namely, ratio of film thickness $\beta=2,5$; inertia parameter $S=0,1,2$; supply radius $r_{o}=0.05$, and step radius $r_{l}=0.4$ have been taken from experimental results of Coombs and Dowson (1964). The results obtained for pressure and load capacity for Newtonian as well as pseudoplastic lubricants are compared with the experimental results of Coombs and Dowson (1964) and established theoretical results of Singh et al. (2011b).

Figure (2) shows the variation of film pressure along the radial direction for step ratio $\beta=2.18$ and values of roughness parameter (c), coefficient of pseudoplasticity $(\kappa)$ and inertia parameter $(S)$. To maintain the graphical clarity, the results for radial and circumferential roughness have been presented as separate figures. In both the case of radial and circumferential roughness patterns, the dimensionless pressure for $c=0$ is same as obtained by Singh et al. (2011b) for each value of $S$, which validates the present results for smooth surfaces. It is, further, observed that radial roughness lowers the dimensionless values of film pressure, while the circumferential roughness increases the dimensionless pressure. In case of the radial roughness patterns $(c=0.0005)$, the pressure is less than that for $c=0$ for Newtonian as well as nonNewtonian lubricants, and the trend holds for each value of $S$, whereas reversed results are obtained for circumferential roughness patterns. Further, in comparison to smooth surface, the theoretical values of pressure obtained for rough surfaces with radial roughness are closer to the experimental values. Pressure for radial roughness $(c=0.0005)$ and nonNewtonian lubricants $\left(\kappa=5.65 \times 10^{-6}\right)$ are more close to the experimental results of Coombs and Dowson (1964) than the earlier theoretical results (Singh et al. 2011b).

In Fig. (3), similar variation of profile of dimensionless pressure has been obtained and compared with established theoretical and experimental results for step ratio $\beta=1.54$. This establishes the sustainability of present results of film pressure for surface roughness. Furthermore, all the figures for pressure also shows that the effects of surface roughness and non-Newtonian pseudoplastic lubricants are more significant at higher values of inertia parameter $S$ 


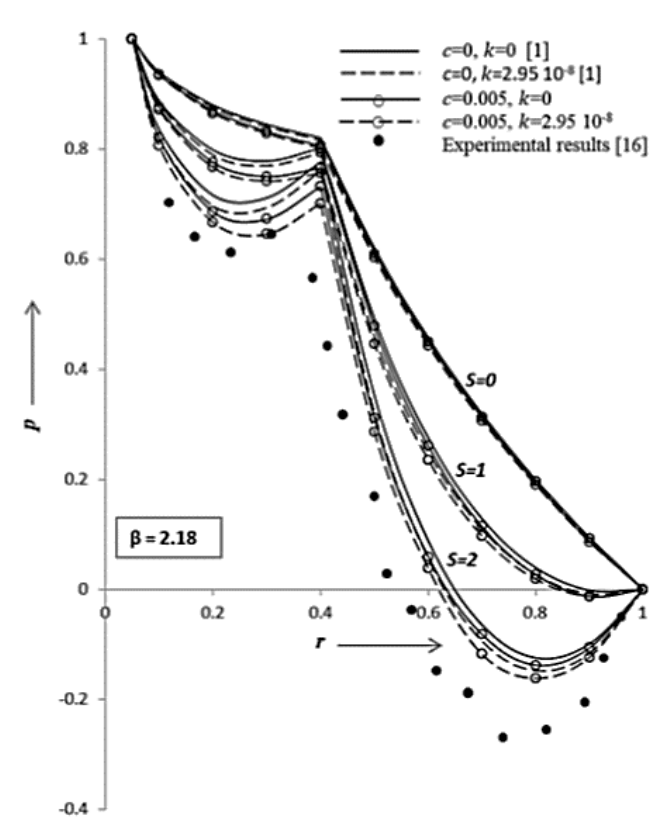

(a)

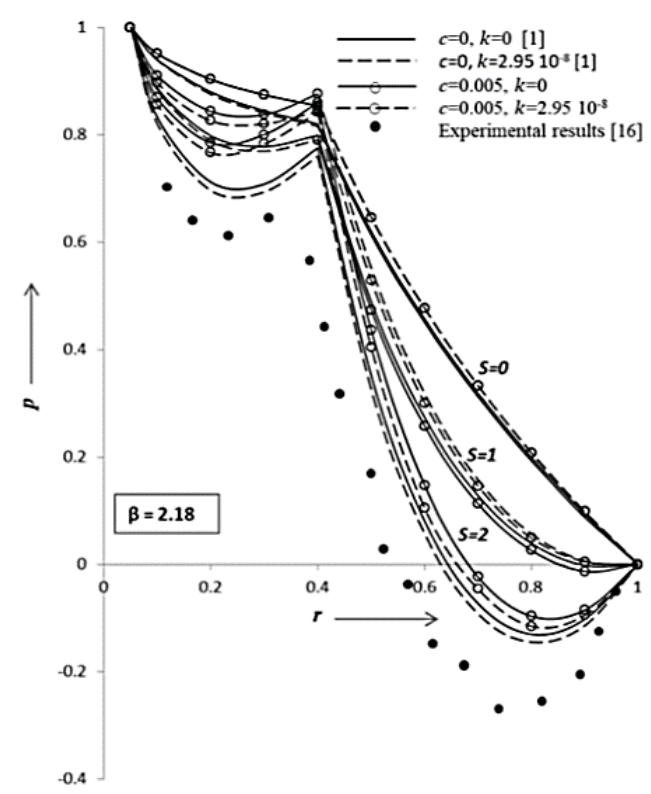

(b)

Fig. 2. Variation of film pressure with radius for inertia $S=0,1,2$ and roughness parameter $(c)$. (a) Radial Roughness (b) Circumferential Roughness.

Figure (4) shows variation of load capacity with respect to the step position $r_{l}$ for different values of $c, \kappa$ and $S$. In case of radial roughness $(c=0.0005)$, the load capacity is less than that for smooth surfaces in both the cases of Newtonian and non-Newtonian lubricants, and the trend of variations sustain for each value of $S$. In case of circumferential roughness, the results are reversed and sustain for variation in other operating and fluid parameters. Furthermore, it can be clearly observed from the figures that higher is the value of inertia parameter $S$, more significant are the effects of surface roughness and non-Newtonian lubricants.

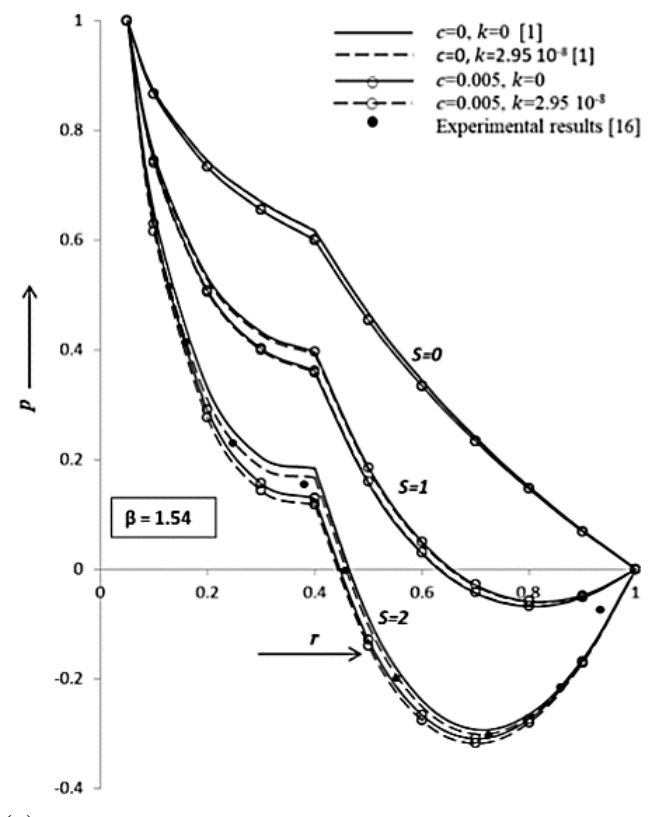

(a)

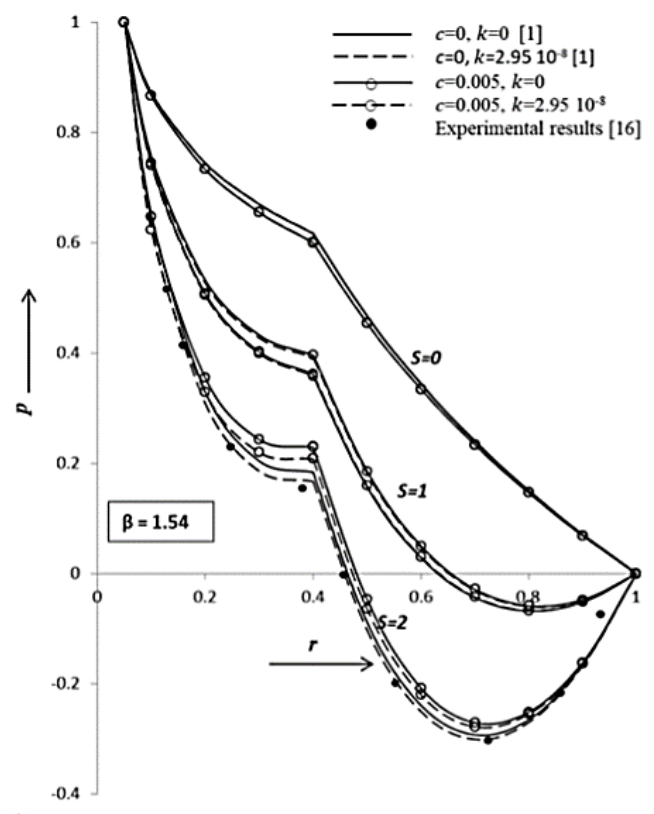

(b)

Fig. 3. Variation of film pressure with radius for values of inertia $S=0,1,2$ and roughness parameter (c). (a) Radial Roughness (b) Circumferential Roughness.

In order to observe precisely the influence of amount and patterns of surface roughness on the load capacity, Fig. (5) has been produced. This figure shows variation of non-dimensional load carrying capacity with respect to roughness parameter $\mathrm{c}$ for values of $S$ and $\kappa$, and a typical value of step parameter $r_{l}=0.4$. Results for both the type of radial and circumferential roughness patterns are plotted simultaneously. It is easy to observe that load capacity decreases with the increase of radial roughness, and increases with the increase of circumferential roughness. The variations sustain 


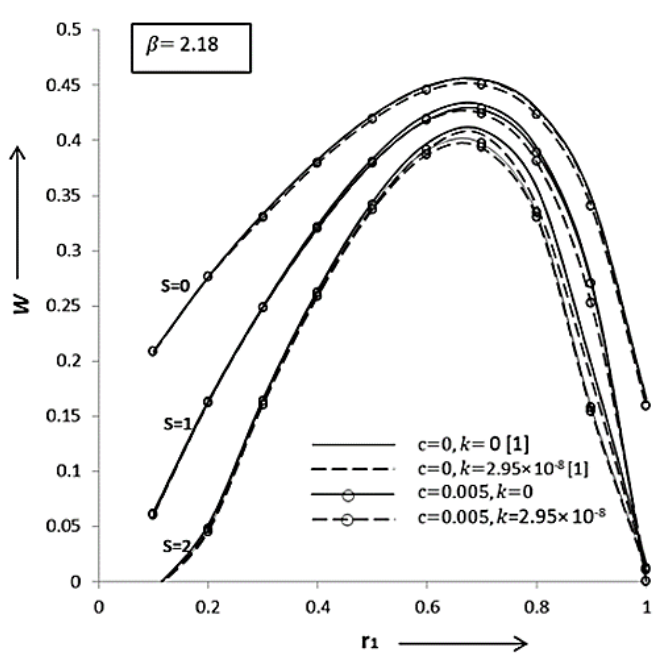

(a)

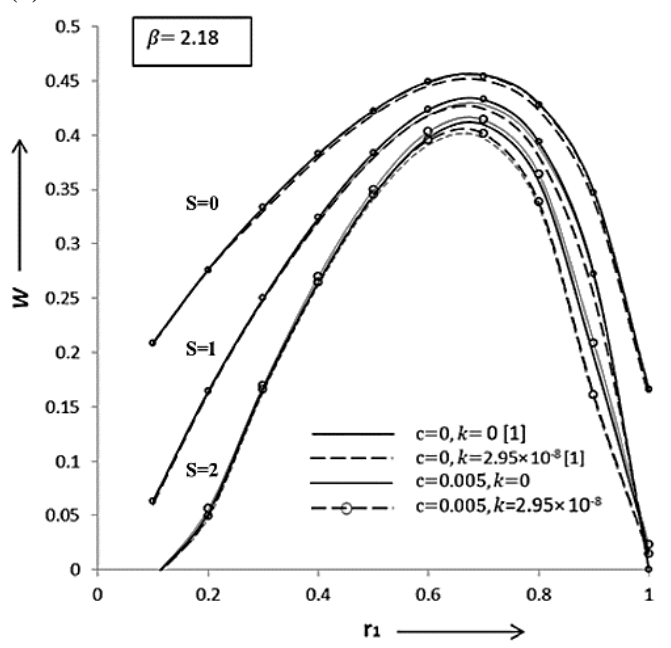

(b)

Fig. 4. Variation of load capacity with step parameter $\left(r_{I}\right)$ for different values of roughness

$(c)$, inertia parameter $(S)$ and coefficient of pseudoplasticity ( ( ) . (a) Radial Roughness (b) Circumferential Roughness.

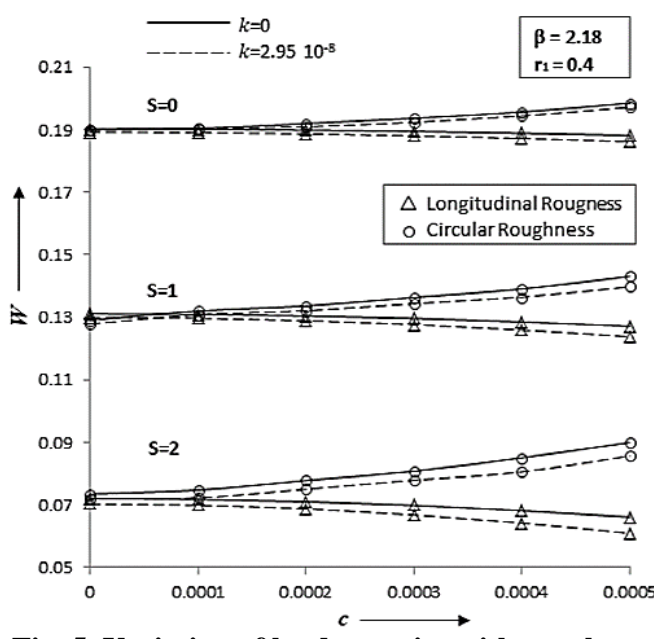

Fig. 5. Variation of load capacity with roughness parameter $(c)$ for $S=0,1,2$ and coefficient of pseudoplasticity $(\kappa)$. ( $\Delta$-Radial Roughness, $\bigcirc$ Circumferential Roughness. their nature for Newtonian as well as pseudoplastic lubricants at each value of inertia parameter S. It is also clearly observed that surface roughness has more significant influence on load capacity for higher values of inertia parameter $S$ because high inertia causes faster discharge of lubricant.

Hence, as a result of simultaneous influences of inertia, surface roughness, non-Newtonian nature of lubricant, the film pressure and load capacity show too significant amount of variation to be ignored in the optimization and modeling of a hydrostatic thrust bearing.

\subsection{Physical Interpretation of Effects of} Roughness, Lubricant and Inertia

\subsection{Effect of Roughness:}

The variation in film pressure due to nature of roughness is physically consistent because in case of radial roughness, asperity ridges and valleys run along the direction of fluid flow. On interaction of fluid with a ridge, fluid tends to flows around the ridge which gives rise to a local pressure drop at a radial point which results into lower load capacity. In case of circumferential roughness, ridges and valleys run in perpendicular to the direction of radial flow, which restricts the available area for flow and diminishes the lubricant flow. This causes an increase in film pressure and, thereby, an increase in load capacity.

\subsection{Effect of Lubricant:}

Effect of inertia: The pseudoplastic fluids have lower viscosity than the Newtonian fluids, due to which the pseudoplastic lubricants cause faster flow and, thereby, lower film pressure and less load capacity.

\subsection{Effect of Inertia:}

Inertia of lubricant is the most important factor influencing the performance characteristics of a hydrostatic thrust bearing. Higher inertia causes faster flow rate of lubricant which causes significant drop in film pressure. Lower film pressure results into lower load capacity of the bearing.

\section{CONCLUSIONS}

Combined effects of surface roughness, nonNewtonian pseudoplastic lubricants and lubricant inertia on the steady performance of hydrostatic thrust bearings, neglecting the radial inertia of lubricant and cavitation effects, have been presented. Rabinowitsch fluid model for non-Newtonian nature of the fluid, Christensen theory for surface roughness and averaged inertia method to derive pressure gradient were used in the analysis, based on which, following conclusions are drawn -

1. In comparison with smooth surfaces, dimensionless film pressure and load capacity is lower for radial roughness and higher for circumferential roughness patterns.

2. With increase of amount of roughness, load carrying capacity decreases for radial roughness 
U. P. Singh / JAFM, Vol. 13, No. 4, pp. 1339-1347, 2020.

and increases for circumferential roughness.

3. In comparison with Newtonian lubricants, dimensionless pressure and load is lower for pseudoplastic lubricants, which is also the established result (Singh, Gupta, and Kapur 2011b).

4. Effects of surface roughness and nonNewtonian lubricants become more significant for larger values of inertia parameter, that is, for larger bearing radius and higher operating speed.

Hence, the present analysis is expected to be helpful for better bearing designs.

\section{Appendix A}

The vector form of the Navier Stokes equation is given as:

$\rho \frac{D \mathbf{q}}{D \bar{t}}=\rho \boldsymbol{B}-\nabla \bar{p}+\nabla \cdot \tau$

where $\mathrm{q}=(\bar{u}, \bar{v}, \bar{w})$ is velocity of the fluid, $\mathbf{B}=\left(\bar{B}_{x}\right.$, $\left.\bar{B}_{y}, \bar{B}_{z}\right)$ is body force per unit mass and $\bar{p}=\bar{p}(\mathrm{x}, \mathrm{y}, z)$ is film pressure, $\rho$ is fluid density and $\tau$ is stress tensor.

Equation of continuity for fluid flow is given as:

$\frac{\partial \rho}{\partial \bar{t}}+\nabla \cdot(\rho \mathrm{q})=0$

In absence of external body forces, the Navier-Stoke Eqs. (24) for incompressible fluids can be represented in cylindrical polar coordinate system as:

$\rho\left(\bar{u} \frac{\partial \bar{u}}{\partial \bar{r}}+\frac{\bar{v}}{\bar{r}} \frac{\partial \bar{u}}{\partial \theta}+\bar{w} \frac{\partial \bar{u}}{\partial \bar{z}}-\frac{\bar{v}^{2}}{\bar{r}}\right)=-\frac{\partial \bar{p}}{\partial \bar{r}}$

$+\left(\frac{\partial \bar{\tau}_{r r}}{\partial \bar{r}}+\frac{1}{\bar{r}} \frac{\partial \bar{\tau}_{r \theta}}{\partial \theta}+\frac{\partial \bar{\tau}_{r z}}{\partial z}\right)$

$\rho\left(\bar{u} \frac{\partial \bar{v}}{\partial \bar{r}}+\frac{\bar{v}}{\bar{r}} \frac{\partial \bar{v}}{\partial \theta}+\bar{w} \frac{\partial \bar{v}}{\partial \bar{z}}+\frac{\bar{u} \bar{v}}{\bar{r}}\right)=-\frac{1}{\bar{r}} \frac{\partial \bar{p}}{\partial \theta}+\left(\frac{\partial \bar{\tau}_{\theta r}}{\partial \bar{r}}+\right.$

$\left.\frac{1}{\bar{r}} \frac{\partial \bar{\tau}_{\theta \theta}}{\partial \theta}+\frac{\partial \bar{\tau}_{\theta z}}{\partial z}\right)$

$\rho\left(\bar{u} \frac{\partial \bar{w}}{\partial \bar{r}}+\frac{\bar{v}}{\bar{r}} \frac{\partial \bar{w}}{\partial \theta}+\bar{w} \frac{\partial \bar{w}}{\partial \bar{z}}\right)=\frac{\partial \bar{p}}{\partial \bar{z}}+\left(\frac{\partial \bar{\tau}_{z r}}{\partial \bar{r}}+\frac{1}{\bar{r}} \frac{\partial \bar{\tau}_{z \theta}}{\partial \theta}+\right.$

$\left.\frac{\partial \bar{\tau}_{z z}}{\partial \bar{z}}\right)$

The equation of continuity (25), in this case, can be written as:

$\frac{1}{\bar{r}} \frac{\partial}{\partial \bar{r}}(\bar{r} \bar{u})+\frac{1}{\bar{r}} \frac{\partial \bar{v}}{\partial \theta}+\frac{\partial \bar{w}}{\partial \bar{z}}=0$

Under the assumptions of thin film lubrication (Cameron 1976) and symmetry of flow applicable to circular bearings, Eqs. (26-29) can be re-written as:

$-\frac{\rho \bar{v}^{2}}{\bar{r}}=-\frac{\partial \bar{p}}{\partial \bar{r}}+\frac{\partial \bar{\tau}_{r z}}{\partial \bar{z}}$

$0=\frac{\partial \bar{\tau}_{\theta z}}{\partial \bar{z}}$

$0=\frac{\partial \bar{p}}{\partial \bar{z}}$

$\frac{1}{\bar{r}} \frac{\partial}{\partial \bar{r}}(\bar{r} \bar{u})+\frac{\partial \bar{w}}{\partial \bar{z}}=0$

System of Eqs. (30-33) have to be solved under the conditions $\bar{u}=0, \bar{w}=0$ at $\bar{z}=0, \bar{h} ; \bar{v}=0$ at $\bar{z}=0$;
$\bar{v}=\mathrm{R} \Omega$ at $\bar{z}=\overline{\mathrm{h}} ; \bar{p}=\mathrm{p}_{\mathrm{o}}$ at $\bar{r}=\mathrm{r}_{\mathrm{o}}$ and $\bar{p}=0$ at $\bar{r}=\mathrm{R}$.

Now dimensionless form (2-5) can be easily obtained with the dimensionless quantities described in nomenclature.

\section{APPENDIX B}

Stochastic form of $I$ is :

$\mathrm{E}(I)=\mathrm{E}\left(-\frac{20}{9} S r+\frac{\partial p}{\partial r}\right)=-\frac{20}{9} S r+\frac{\partial E(p)}{\partial r}$

where, $E\left(\frac{\partial p}{\partial r}\right)=\frac{\partial E(p)}{\partial r}$, (Christensen 1969).

Further,

$$
\begin{aligned}
\mathrm{E}\left(I^{3}\right)=\mathrm{E}\left[\left(-\frac{20}{9} S r+\frac{\partial p}{\partial r}\right)^{3}\right] & \mathrm{E}\left[-\left(\frac{20}{9} S r\right)^{3}+3\left(\frac{20}{9} S r\right)^{2}\left(\frac{\partial p}{\partial r}\right)\right. \\
& \left.-3\left(\frac{20}{9} S r\right)\left(\frac{\partial p}{\partial r}\right)^{2}+\left(\frac{\partial p}{\partial r}\right)^{3}\right] \\
=-\left(\frac{20}{9} S r\right)^{3}+3\left(\frac{20}{9} S r\right)^{2} \frac{\partial \mathrm{E}(p)}{\partial r} & -3\left(\frac{20}{9} S r\right)\left(\frac{\partial \mathrm{E}(p)}{\partial r}\right)^{2}+\left(\frac{\partial \mathrm{E}(p)}{\partial r}\right)^{3}
\end{aligned}
$$

\section{APPENDIX C}

\section{CASE I: Radial Roughness}

Continuity of pressure, Eqs. (16-17), gives the expression for flow rate as:

$$
\begin{aligned}
& Q=\left(\frac{\phi}{2 A}+\sqrt{\left(\frac{\phi}{2 A}\right)^{2}+\left(\frac{B}{3 A}\right)^{2}}\right)^{1 / 3} \\
& -\frac{B}{3 A}\left(\frac{\phi}{2 A}+\sqrt{\left(\frac{\phi}{2 A}\right)^{2}+\left(\frac{B}{3 A}\right)^{2}}\right)^{-1 / 3}
\end{aligned}
$$

where, $\varphi=1+\frac{9}{10} \mathrm{~S}\left(1-r_{0}^{2}\right)$ and,

$$
\begin{gathered}
A=\frac{2187}{495} \frac{\mu^{3}}{\pi^{3}} \alpha \\
\times\left[\frac{45 c^{4} h \beta+330 c^{2} h^{3}+729 h^{5} \beta^{5}}{\left(c^{2} h \beta+h^{3} \beta^{3}\right)^{4}}\right. \\
\times\left(\frac{1}{r_{1}^{2}}-\frac{1}{r_{0}^{2}}\right) \\
\quad-\frac{45 c^{4} h+330 c^{2} h^{3}+729 h^{5}}{\left(c^{2} h+h^{3}\right)^{4}} \\
\left.\quad \times\left(\frac{1}{r_{1}^{2}}-1\right)\right] \\
B=\frac{18 \mu}{\pi}\left[\frac{1}{c^{2} h \beta+h^{3} \beta^{3}} \log \left(\frac{r_{1}}{r_{0}}\right)\right. \\
-\frac{1}{c^{2} h+h^{3}} \log r_{1}
\end{gathered}
$$

CASE II: Circumferential Roughness

Continuity of pressure, Eqs. (18-23), gives the expression for flow rate as:

$Q=\left(\frac{\phi}{2 A}+\sqrt{\left(\frac{\phi}{2 A}\right)^{2}+\left(\frac{B}{3 A}\right)^{2}}\right)^{1 / 3}$ 


$$
-\frac{B}{3 A}\left(\frac{\phi}{2 A}+\sqrt{\left(\frac{\phi}{2 A}\right)^{2}+\left(\frac{B}{3 A}\right)^{2}}\right)^{-1 / 3}
$$

where, $\varphi=1+\frac{9}{10} \mathrm{~S}\left(1-r_{0}^{2}\right)$ and,

$B=\frac{81}{5} \frac{\mu^{3}}{\pi^{3}} \alpha\left(\frac{A_{1}^{4}}{A_{2}} \frac{\mu^{3}}{\pi^{3}}\left(\frac{1}{r_{1}^{2}}-\frac{1}{r_{0}^{2}}\right)\right.$

$\frac{B_{1}^{4}}{B_{2}}\left(\frac{1}{r_{1}^{2}}-1\right)$

$B=\frac{6 \mu}{\pi}\left(A_{1} \log \left(\frac{r_{1}}{r_{0}}\right)-B_{1} \log r_{1}\right)$

\section{REFERENCES}

Bakker, O. J. and R. A. J. van Ostayen (2010). Recess Depth Optimization for Rotating, Annular, and Circular Recess Hydrostatic Thrust Bearings. Journal of Tribology 132(1), $1-7$.

Bassani, R. and P. Piccigallo (1992). Hydrostatic lubrication. Amsterdam: Elsevier.

Bhatt, S. S., A. Medhavi, R. S. Gupta and U. P. Singh (2017). Effects of Heat Transfer during Peristaltic Transport in Nonuniform Channel with Permeable Walls. Journal of Heat Transfer 139(4), 014502,1-5.

Bourging, P. and B. Gay (1984). Determination of the load capacity of finite width journal bearing by finite element method in the case of a nonNewtonian lubricant. Journal of Tribology 106, 285-290.

Cameron, A. (1976). Basic lubrication theory. Chichester, UK.: Ellis Horwood.

Christensen, H. (1969). Stochastic Models for Hydrodynamic Lubrication of Rough Surfaces. Proceedings of the Institution of Mechanical Engineers 184(55), 1013-1026.

Coombs, J. A. and D. Dowson (1964). An experimental investigation of the effects of lubricant inertia in a hydrostatic thrust bearing. Proceedings of the Institution of Mechanical Engineers, London 179 (Paper, 96-108.

Hamrock, B. J., S. R. Schmid and B. O. Jacobson (2004). Fundamentals of fluid film lubrication. CRC Press.

Hashimoto, H. and S. Wada (1986). The Effects of Fluid Inertia Forces in Parallel Circular Squeeze Film Bearings Lubricated with Pseudo-Plastic Fluids. Journal of Tribology 108(2), 222-227.

Hayashi, H. and S. Wada (1974). Hydrodynamic Lubrication of Journal Bearings by PseudoPlastic Lubricants (Part 3, Theoretical Analysis Considering Effects of Correlation). JSME 17(109), 967-974.

Kapur, V. K. and K. Verma (1979). The simultaneous effects of inertia and temperature on the performance of a hydrostatic thrust bearing. Wear 54(1), 113-122.
Lin, J. R. (1999). Static and dynamic characteristics of externally pressurized circular step thrust bearings lubricated with couple stress fluids. Tribology International 32(4), 207-217.

Lin, J. R. (2000). Surface roughness effect on the dynamic stiffness and damping characteristics of compensated hydrostatic thrust bearings. International Journal of Machine Tools and Manufacture 40(11), 1671-1679.

Lin, J. R. (2012). Non-Newtonian squeeze film characteristics between parallel annular disks: Rabinowitsch fluid model. Tribology International 52, 190-194.

Lin, J. R. (2001). Non-Newtonian effects on the dynamic characteristics of one dimensional slider bearings: Rabinowitsch model. Tribology Letters 10(4), 237-243.

Peterson, J., W. E. Finn and D. W. Dareing (1994). Non-Newtonian temperature and pressure effects of a lubricant slurry in rotating hydrostatic step bearing. Tribology Transactions 37(4), 857-863.

Prakash, J. and K. Tiwari (1985). Effect of surface roughness on the squeeze film between rotating porous annular discs with arbitrary porous wall thickness. International Journal of Mechanical Sciences 27(3), 135-144.

Sawano, H., Y. Nakamura, H. Yoshioka and H. Shinno (2015). High performance hydrostatic bearing using a variable inherent restrictor with a thin metal plate. Precision Engineering 41, 78-85.

Sharma, S. C., S. C. Jain and D. K. Bharuka (2002). Influence of recess shape on the performance of a capillary compensated circular thrust pad hydrostatic bearing. Tribology International 35(6), 347-356.

Singh, P., B. D. Gupta and V. K. Kapur (1983). Design criteria for stepped thrust bearings. Wear 89(1), 41-55.

Singh, P., B. D. Gupta and V. K. Kapur (1993). Optimization of corrugated thrust bearing characteristics. Wear 167(2), 109-120.

Singh, U. P., A. Medhavi, R. S. Gupta and S. S. Bhatt (2017). Analysis of Peristaltic Transport of Non-Newtonian Fluids Through Nonuniform Tubes: Rabinowitsch Fluid Model. Zeitschrift fur Naturforschung - Section A Journal of Physical Sciences 72(7), 1-8.

Singh, U. P., A. Medhavi, R. S. Gupta and S. S. Bhatt (2018). Theoretical study of heat transfer on peristaltic transport of Non-Newtonian fluid flowing in a channel: Rabinowitsch fluid model. International Journal of Mathematical, Engineering and Management Sciences 3(4), 450-461.

Singh, U. P., R. S. Gupta and V. K. Kapur (2011b). On the steady performance of hydrostatic thrust bearing: Rabinowitsch fluid model. Tribology 
U. P. Singh / JAFM, Vol. 13, No. 4, pp. 1339-1347, 2020.

Transactions 54(5), 723-729.

Singh, U. P., R. S. Gupta and V. K. Kapur (2012a). Non-Newtonian Effects on the Squeeze Film Characteristics between a Sphere and A Flat Plate: Rabinowitsch Model. Advances In Tribology 2012(ArticleID:571036), 1-7.

Singh, U. P., R. S. Gupta and V. K. Kapur (2012b). On the performance of pivoted curved slider bearings: Rabinowitsch fluid model. Tribology in Industry 34(3), 1-7.

Singh, U. P., R. S. Gupta and V. K. Kapur (2012c). On the steady performance of annular hydrostatic thrust bearing: Rabinowitsch fluid model. Journal of Tribology 134(4), 044502:15 .

Singh, U. P., R. S. Gupta and V. K. Kapur (2013a). On the application of Rabinowitsch fluid model on an annular ring hydrostatic thrust bearing. Tribology International 58, 65-70.

Singh, U. P., R. S. Gupta and V. K. Kapur (2013b). On the squeeze film characteristics between a long cylinder and a flat plate: Rabinowitsch model. Proceedings of the Institution of Mechanical Engineers, Part J: Journal of Engineering Tribology 227(1), 34-42.

Singh, U., R. Gupta and V. Kapur (2011a). Effects of inertia in the steady state pressurised flow of a non-Newtonian fluid between two curvilinear surfaces of revolution: Rabinowitsch fluid model. Chemical and Process Engineering 32(4), 333-349.

Spikes, H. A. (1994). The Behaviour of Lubricants in Contacts: Current Understanding and Future Possibilities. Proceedings of the Institution of Mechanical Engineers, Part J: Journal of Engineering Tribology 208(1), 3-15.

Stokes, V. K. (1966). Couple stress in fluids. Phys. Fluids. 9, 1709-1715.

Tian, Z., H. Cao, and Y. Huang (2018). Static characteristics of hydrostatic thrust bearing considering the inertia effect on the region of supply hole. Proceedings of the Institution of Mechanical Engineers, Part J: Journal of Engineering Tribology 233, 188-193.
Wada, S. and H. Hayashi (1971). Hydrodynamic Lubrication of Journal Bearings by PseudoPlastic Lubricants: Part 2, Experimental Studies. Bulletin of JSME 14(69), 279-286.

Walicka, A., E. Walicki, P. Jurczak and J. Falicki (2014). Thrust Bearing with Rough Surfaces Lubricated by an Ellis Fluid. International Journal of Applied Mechanics and Engineering 19(4), 809-822.

Walicka, A., E. Walicki, P. Jurczak and J. Falicki (2017a). Curvilinear squeeze film bearing with porous wall lubricated by a Rabinowitsch fluid. International Journal of Applied Mechanics and Engineering 22(2), 427-441.

Walicka, A., E. Walicki, P. Jurczak and J. Falicki (2017b). Influence of wall porosity and surfaces roughness on the steady performance of an externally pressurized hydrostatic conical bearing lubricated by a Rabinowitsch fluid. International Journal of Applied Mechanics and Engineering 22(3), 717-737.

Xuebing, Y., X. Wanli, L. Lang and H. Zhiquan (2009). Analysis of the Combined Effect of the Surface Roughness and Inertia on the Performance of High-Speed Hydrostatic Thrust Bearing. In Z. Q. e. In: Luo J., Meng Y., Shao T. (Ed.), Advanced Tribology, Berlin, Heidelberg, 197-201. Springer.

Yacout, A. W. (2008). The surfaces roughness effect on the hydrostatic thrust spherical bearings performance. Proceedings of the Asme International Mechanical Enginering Congress and Exposition 2007 9(Pts a-C), 431-47.

Yadav, J. S. and V. K. Kapur (1981). On the viscosity variation with temperature and pressure in thrust bearing. International Journal of Engineering Science 19(2), 269-277.

Younes, Y. K. (1993). A revised design of circular hydrostatic bearings for optimal pumping power. Tribology International 26(3), 195-200.

Zhicheng, P., S. Jingwu, Z. Wenjie, L. Qingming and C. Wei (1993). The dynamic characteristics of hydrostatic bearings. Wear 166(2), 215-220. 